\title{
Natura@economía
}

ISSN 2226-9479 (Versión electrónica)

Website: http://revistas.lamolina.edu.pe/index.php/neu

\section{Análisis bioeconómico del consumo animal en condiciones de pastoreo extensivo de la sierra central del Perú}

\begin{abstract}
Bioeconomic analysis of animal consumption under extensive grazing conditions in the central highlands of Perú
\end{abstract}

\author{
Marco Antonio Gutiérrez Tang ${ }^{1}$; Hugo Ibrahim Luna Astorga ${ }^{2}$
}

${ }^{1}$ Ingeniero Zootecnista, investigador independiente, Lima, Perú.

${ }^{2}$ Universidad Nacional Agraria La Molina, Lima, Perú. Email: hugoluna@lamolina.edu.pe

Recepción: 15/10/2019; Aceptación: 15/12/2019

\section{Resumen}

El objetivo de esta investigación fue estimar el consumo animal óptimo, considerando aspectos biológicos y económicos en condiciones de pastoreo extensivo en la sierra central del Perú. La base de datos se construyó con información proveniente de estudios de perfiles alimentarios de explotaciones ganaderas que permitieron conocer el efecto de la carga animal sobre la dinámica de vegetación y el nivel de remoción de la misma para vacunos. Se aplicó el modelo bioeconómico de Gordon y Schaefer, el enfoque de carga animal segura y máxima de Noy - Meier y el método de Galarza y Collado para la estimación del precio sombra de los recursos naturales. Se estimó que la carga animal óptima expresada en unidad animal mes por hectárea (UAM/ha) fue de 2,26 cuando se empleó los precios de mercado. El nivel de carga óptima tiene asociado un stock de biomasa disponible de 4587,32 $\mathrm{kg}$ de materia seca por hectárea al mes (MS/ha/mes), un nivel de consumo de $970 \mathrm{~kg} \mathrm{MS} / \mathrm{ha} / \mathrm{mes}$ y un nivel de producción de leche de 381,43 litros. Con el precio sombra del forraje, calculado en $\mathrm{S} /$ 0,52 soles por $\mathrm{kg}$ de MS, la carga animal óptima disminuye hasta 2,01 UAM. Los beneficios económicos se redujeron de S/ 224,26 a S/ 154,42 al emplear ambos enfoques de precios.

Palabras clave: Análisis bioeconómico; crecimiento y disponibilidad de forraje; consumo animal; carga animal; maximización de beneficios.

\begin{abstract}
The objective of this research was to estimate the optimal animal consumption, considering biological and economic aspects in conditions of extensive grazing in the central highlands of Peru. The database was constructed with information from studies of food profiles of livestock farms that allowed to know the effect of stocking rate on the dynamics of vegetation and its level of removal for cattle. The bioeconomic model of Gordon and Scheafer, the approach of safe and maximum animal load of Noy Meier and the method of Galarza and Collado were applied to estimate the shadow price of natural resources. It was estimated that the optimal
\end{abstract}

Forma de citar el artículo: Gutiérrea, M.; Luna, H. 2019. Análisis bioeconómico del consumo animal en condiciones de pastoreo extensivo de la sierra central del Perú. Natura@economía 4(2): 59- 69 (2019).

DOI: http://dx.doi.org/10.21704/ne.v4i2.1409

* Autor de correspondencia: Hugo Ibrahim Luna Astorga. Email: hugoluna@lamolina.edu.pe

(C) Facultad de Economía y Planificación, Universidad Nacional Agraria La Molina, Lima, Perú. 
animal load expressed in animal unit month per hectare (AMU/ha) was 2,26 when market prices were used. The optimum stocking rate has an associated stock of available biomass of $4587,32 \mathrm{~kg}$ of dry matter per hectare per month (DM/ha/month), a consumption level of 970 $\mathrm{kg} \mathrm{DM} / \mathrm{ha} /$ month and a milk production level of 381,43 liters. With the shadow price of the forage, calculated at $\mathrm{S} / 0,52$ soles per $\mathrm{kg}$ of $\mathrm{DM}$, the optimal stocking rate decreases to 2,01 AMU. The economic benefits were reduced from S/ 224,26 to S/ 154,42 soles by using both price approaches.

Keywords: Bioeconomic analysis; growth and availability of forage; animal consumption; stocking rate; maximization of benefits.

\section{Introducción}

El Instituto Nacional de Estadística e Informática del Perú señala que el $46,8 \%$ de los pobres y el 70,4\% de los pobres extremos del país, se encuentran localizados en la región sierra del Perú percibiendo ingresos mensuales promedios menores a los 300 soles (INEI, 2019). Las comunidades altoandinas dependen exclusivamente de los recursos naturales y ecosistemas circundantes, no solo desde el punto de vista productivo sino también ecológico, económico y ambiental. En la región andina, el área ocupada por los pastizales naturales abarca cerca de 18 millones de hectáreas (MINAM, 2018), de acuerdo al III y IV Censo Nacional Agropecuario (INEI, 1994; INEI, 2012), cubren entre el 12 a $14 \%$ del territorio nacional, sobrepasando el uso potencial del suelo de 8\% (ONERN, 1985). Estas praderas generan servicios ambientales (secuestro de carbono, regulación hídrica y control de inundaciones, entre otros), cumplen funciones de conservación de la biodiversidad y protección de hábitats (MEA, 2005; TEEB, 2010), pero además son fuente de alimento para más del $80 \%$ de la población ganadera del país. La producción animal en dichas condiciones no está libre de limitaciones, debido a una inapropiada gestión del recurso forrajero por parte de los agentes económicos localizados en ellas, los cuales desconocen el valor que los servicios ecosistémicos agregan a la producción.

La biomasa forrajera o la producción de forraje se definen como el peso de las formas de vida vegetales presentes en un momento determinado, la cual es influenciada por el tipo de pradera, la temperatura y principalmente por la humedad. La biomasa se expresa internacionalmente en Kilogramos (materia seca o material fresco) por hectárea por año o en alguna otra unidad de tiempo (ASRM,
1962). La biomasa forrajera es un indicador importante de los procesos ecológicos y de la gestión de la vegetación, su estimación solo considera a la parte aérea que está por encima del suelo y comúnmente disponible para herbívoros grandes. La producción de forraje ( $\mathrm{kg} / \mathrm{MS} / \mathrm{ha} / \mathrm{año})$ es la suma de las tasas de crecimiento $(\mathrm{kg} / \mathrm{MS} / \mathrm{ha} / \mathrm{día})$ multiplicado por el número de días del mes, a lo largo del año.

La degradación de un ecosistema pastoril ocurre cuando la biomasa forrajera es utilizada sin respetar las tasas naturales de reposición, es decir, ocurre el sobrepastoreo o sobre carga animal. En nuestro país, el procedimiento tradicional para estimar la capacidad de carga animal o el número de animales que soporta el sistema sin causar deterioro ni retrogresión, se basa muchas veces solo en cálculos de los cambios en la disponibilidad de forraje mes a mes, sin tomar en cuenta la tasa de crecimiento del forraje, el nivel de uso de la vegetación ni el aporte energético de este (Ñaupari y Flores, 1996), es decir, existen deficiencias en las técnicas de medición del recurso para una óptima estimación.

De acuerdo con las características ecológicas de la puna, se calcula que se puede utilizar de $50 \%$ a $80 \%$ de la producción anual de forrajes, sin producir efectos negativos en el suelo, pero en la puna seca no debe de superar los 70\% como máximo (Flórez y Malpartida, 1987). Un experimento realizado sobre una pastura cultivada con una asociación de Dactylis-Trebol y una pradera nativa con Festuca-Calamagrostis determinó tasas de crecimiento de la biomasa del forraje de $11,7 \mathrm{~kg} / \mathrm{MS} / \mathrm{ha} /$ día (351 kg/MS/ha/mes) y 7,7 kg MS/ha/día (231 kg MS/ha/mes) respectivamente, para una explotación ganadera sobre los 4000 metros de altitud (Flores, 2006). En Puno, 
en una pradera cultivada se registraron producciones de forraje de hasta $952 \mathrm{~kg} /$ ha, resultado superior a los $180 \mathrm{~kg} / \mathrm{ha}$ producidos en un pastizal natural (Flórez y Bryant, 1990).

Bajo condiciones de secano, no parece existir diferencias entre las tasas de crecimiento del forraje de una pradera cultivada y una natural, encontrándose producciones promedias que no superan los $10 \mathrm{~kg} / \mathrm{MS} / \mathrm{ha} /$ día $(300 \mathrm{~kg} / \mathrm{ha} / \mathrm{mes})$. Sin embargo, cuando la condición de precipitación y temperatura fueron óptimas, las tasas se diferenciaron en el orden de $8 \mathrm{~kg}$ $\mathrm{MS} /$ día (240 kg/mes) para la pradera nativa y de $50 \mathrm{~kg} \mathrm{MS} /$ día (1500 kg/mes) para la pastura cultivada (Flores, 2006). Naupari (2000), menciona que en la época lluviosa las diferencias entre la producción de forraje de una pradera cultivada y una natural es de 2,3 veces más, y puede superar los $8000 \mathrm{~kg}$ $\mathrm{MS} / \mathrm{ha}$ en un periodo de siete meses.

Perfiles alimentarios desarrollados para diversas explotaciones ganaderas en condiciones de sierra central encontraron que el crecimiento de la vegetación depende de dos factores principales: el primero, de las condiciones climáticas apropiadas para la producción de la biomasa forrajera y segundo, que a mayor sea la carga animal a la que se presiona la pastura el crecimiento y la disponibilidad se verán afectados negativamente (Ñaupari, 2000; Candelario, 2005; Avalos, 2006; Esponda, 2007). Por ende, la forma como gestionan los pastizales los pobladores que dependen de ellos es un factor importante para su conservación o degradación, esto nos lleva a analizar cuáles pueden ser los factores que ocasionan el sobre uso de algunos recursos naturales como es el caso de los pastizales.

Como lo menciona Bromley (1991) cuando se poseen derechos de propiedad completos sobre los recursos naturales se puede realizar una óptima asignación de estos, ya que se puede internalizar las externalidades y se generan los incentivos necesarios para que el comportamiento racional de los agentes los impulse a su uso eficiente. Un agente posee derechos de propiedad completos sobre un recurso cuando posee los derechos de uso y de exclusión, es decir puede usar el recurso como mejor le convenga asumiendo los beneficios y las obligaciones que de sus decisiones se desprenden, y puede excluir a terceros de su uso (Canavese, 2007). Los pastizales y más precisamente el recurso forrajero se caracterizan por ser un recurso catalogado como de uso común los cuales presentan características de ser no excluibles pero rivales en su extracción, es decir, pueden ser utilizados o consumidos por cualquier agente económico sin ningún tipo de limitaciones debido a la ausencia de derechos de propiedad. Estas dos características (no excluibles y rivales) en simultáneo hacen que estén inmersos en una situación conocida como la tragedia de los comunes (Ward, 2006).

La tragedia de los comunes descrita por Hardin (1968) plantea una situación que se presenta cuando existe un recurso común o comunal, es decir no existen restricciones al acceso, su uso ocasiona costos cada vez mayores a los agentes que lo explotan (rivalidad), de igual forma, los agentes económicos actuando racionalmente pero individualmente en su afán por maximizar sus beneficios terminan por destruir el recurso (Caffera, 2018).

Ostrom (2011), menciona que "es importante que los agentes o usuarios locales de un recurso que tenga características de bien de uso común tengan acuerdos claros sobre reglas de definición de límites, mecanismos de solución de conflictos, planes de monitoreo, sanciones graduadas apropiadas y sus propias reglas relacionadas con el aprovechamiento del recurso a fin de evitar caer en la trampa de la tragedia".

La clasificación de los recursos naturales en renovables y no renovables nos proporcionan tres tipos de modelos de análisis: para los recursos renovables se tienen los llamados modelos de pesca y modelos de bosques, y para los no renovables los modelos de minas (Romero, 1994; Riera et al., 2005; Maldonado, 2008). A pesar de las actividades de las cuales derivan los nombres de los modelos, estos deben de verse como propuestas de análisis más generales y no excluyen a que otros recursos naturales se puedan ajustar a la metodología de los modelos mencionados (Romero, 1994; Galarza, 2004) como por ejemplo los pastizales. 
Una alternativa para evitar la degradación de estos ecosistemas y conducir un sistema de producción racional es la implementación de planes de manejo sostenibles que consideren factores de producción biológicos como económicos, para alcanzar niveles de producción óptimos. Por ende, basándose en los modelos de recursos renovables (modelos bioeconomicos), la solución óptima desde un punto de vista económico resultará de la combinación de información de origen biológico y de ciertas variables económicas para determinar el momento óptimo de uso del recurso o la combinación de alternativas que generen los mayores beneficios económicos (Conrad, 2010; Ward, 2006). $\mathrm{n}$ tal sentido, el objetivo de la presente investigación fue estimar el consumo animal óptimo, considerando aspectos biológicos y económicos, que permita la sostenibilidad del recurso y la maximización de los beneficios de los involucrados en la actividad ganadera.

\section{Materiales y métodos}

\section{Base de datos}

La información empleada para este estudio fue construida a partir de los estudios de "Perfiles Alimentarios" realizados por Esponda (2007), Avalos (2006), Candelario (2005) y Naupari (2000); las variables consideradas fueron: consumo animal (Yt), crecimiento de la biomasa forrajera $(\mathrm{Ct})$, biomasa forrajera $(\mathrm{Zt})$, carga animal $(\mathrm{Ca})$ y producción de leche (L). La información es del tipo mensual para el periodo 19962004. Sin embargo, debido a la ausencia de información durante meses no evaluados de cada respectivo estudio se decidió trabajar la información como datos del tipo de corte transversal.

\section{Función de crecimiento $(\mathbf{C t})$}

El crecimiento de una pastura, se estimó mediante la función logística (Verhulst, 1938) la cual es empleada en varios estudios para medir el crecimiento de las poblaciones biológicas de diversos tipos de recursos naturales (Gordon, 1954; Schaefer, 1954; Seijo et al., 1997; Ritten et al., 2010). Se realizó un análisis de regresión por el método de mínimos cuadrados ordinarios (MCO), para poder estimar la tasa del crecimiento (r) y la capacidad de carga $(\mathrm{K})$ de una pastura. Al modelar econométricamente la función logística, esta quedo expresada de la siguiente forma matemática sin intercepto:

$$
C t=a Z t+b Z t^{2}
$$

Donde "a y b" son los estimadores de las variables.

Donde: $\mathrm{a}=\mathrm{r}$ y $\mathrm{b}=-\mathrm{r} / \mathrm{K}$

Finalmente, la función logística se expresa de la siguiente manera:

$$
C t=r Z t(1-Z t / K)
$$

\section{Función de consumo animal (Yt)}

El consumo animal es una variable que depende del stock de la biomasa disponible (Zt) y del número de animales que la aprovechan $(\mathrm{Ca})$. Tomando en cuenta la ecuación del equilibrio en estado estacionario (Gordon, 1954; Schaefer, 1954; Seijo et al., 1997; Galarza, 2004; Ritten et al., 2010), donde la extracción o consumo animal en el largo plazo es igual al crecimiento del recurso, tenemos la siguiente expresión matemática:

$$
\begin{gathered}
C t\left(Z t, Z t^{2}\right)=Y t(Z t, C a) \\
a Z t+b Z t^{2}=q Z t C a
\end{gathered}
$$

Donde se puede conocer que:

$$
C a=a / q+b / q Z t \text { y además } t=q / b C a-a / b
$$

Finalmente introduciendo $\mathrm{Zt}$ en $\mathrm{Yt}(\mathrm{Zt}, \mathrm{Ca})$, tenemos:

$$
Y t=-a q / b C a+q^{2} / b C a^{2}
$$

Donde se puede apreciar que el consumo (Yt) depende de la carga animal y a la vez de los parámetros de la función del crecimiento de la biomasa y de un factor "q". Para 
obtener "q", se estimó econométricamente por $\mathrm{MCO}$, el consumo ( $\left.\mathrm{Yt}_{\mathrm{c}}\right)$ quedando una función de la siguiente forma:

$$
Y t c=x C a+w C a^{2}
$$

Se iguala el coeficiente del término lineal para calcular el valor de "q" tal como se expresa en el siguiente paso:

$$
\mathrm{x}=-\mathrm{aq} / \mathrm{b}
$$

Donde “q” es igual a:

$$
\mathrm{q}=\mathrm{xb} /(-\mathrm{a})
$$

Con lo cual el valor de "q" se ingresa en la función Yt es decir: $Y t z=f\left(Z t, Z t^{2}\right)$, por lo tanto, el nivel de consumo de biomasa puede quedar expresado de la siguiente forma:

$$
Y t z=c Z t+d Z t^{2}
$$

Donde $\quad Y t z\left(Z t, Z t^{2}\right)=C t\left(Z t, Z t^{2}\right), \quad$ nos indicaría el nivel actual del consumo animal y su respectivo nivel de carga animal asociada.

\section{Función de producción de leche $(\mathrm{L})$}

La producción de leche, litros/mes/hectárea, se calculó en base a la información de los estudios de Perfiles Alimentarios revisados, considerando las siguientes variables: producción promedio de leche por animal día, número de animales por hectárea y días de cada mes.

Con los litros de leche (lt/mes/ha), se estimó una función de producción que depende del nivel consumo animal, donde también el consumo depende a su vez del nivel de carga animal.

$$
\begin{aligned}
& \mathrm{L}=\mathrm{f}(\mathrm{Yt}) \\
& \mathrm{L}=\delta \mathrm{Yt} \\
& L=\delta Y t=-(\delta a q / b) \mathrm{Ca}+\left(\delta q^{2} / \mathrm{b}\right) \mathrm{Ca}^{2}
\end{aligned}
$$

Finalmente, la producción de leche queda con la siguiente expresión matemática:

$$
L=\alpha C a+\beta C a^{2}
$$

Ecuación propuesta por Jones and Sandland (1974), donde:

$$
\alpha=-\delta a q / b \quad \text { y } \beta=\delta q^{2} / b
$$

\section{Función de ingresos y costos}

Los ingresos fueron calculados mediante la multiplicación de la cantidad de leche producida por el precio de mercado, considerado $\mathrm{S} / 1,50$ por litro de leche.

Función de Ingresos Totales: $I T=P_{L}{ }^{*} L$

Para el caso de los costos totales, al carecer de esta información en los estudios y ante la imposibilidad de conseguirlos, se optó por tomar una proporción de los ingresos tomando como referencia la estructura del estudio Presupuesto y Rentabilidad del Módulo Lechero: Caso Ayaracra publicado dentro del Reporte Científico titulado "Comportamiento Nutricional, Perfil Alimentario y Economía de la Producción Lechera en Praderas Cultivadas: Caso Pasco" (Flores et al., 2005). Se realizó la regresión con el nivel de carga animal respectivo, tomando en cuenta la ecuación del modelo bio-económico base: $C T=f(C a)$.

$$
\text { Función de Costos Totales: } C T=\theta C a
$$

\section{Función de beneficios}

Los beneficios de la actividad ganadera al pastoreo fueron estimados con la siguiente función: $\pi=I T-C T$

Donde los beneficios están en función del nivel de la carga animal.

$\pi=P_{L}^{*} L-\theta C a$, por lo tanto

$\pi=P_{L} *\left(\alpha C a+\beta C a^{2}\right)-\theta C a$ 


\section{Estimación de los óptimos}

En base a la propiedad de maximización de los beneficios se estimaron los óptimos de carga animal $\left(\mathrm{Ca}^{*}\right)$, biomasa disponible $\left(\mathrm{Zt}^{*}\right)$, consumo animal ( $\left.\mathrm{Yt}^{*}\right)$, producción de leche $\left(\mathrm{L}^{*}\right)$ y beneficios óptimos $\left(\pi^{*}\right)$, donde:

$\partial \pi / \partial C a=\partial I T / \partial C a-\partial C T / \partial C a=0$

Dónde: Ca es igual al óptimo $\mathrm{Ca}^{*} \mathrm{y}$, además:

$$
\begin{gathered}
\mathrm{Zt}^{*}=\mathrm{q} / \mathrm{b} \mathrm{Ca}-\mathrm{a} / \mathrm{b} \\
Y t^{*}=-(a q / b) C a^{*}+\left(q^{2} / b\right) C a^{* 2} \\
L^{*}=\alpha C a^{*}+\beta C a^{* 2} \\
\pi^{*}=P_{L}^{*}\left(\alpha C a^{*+} \beta C a^{* 2}\right)-\theta C a^{*}
\end{gathered}
$$

\section{Resultados y discusión}

\section{Función de crecimiento}

La función de crecimiento de la biomasa estimada fue significativa $(p<00,5)$, a partir de los estimadores se calculó la tasa intrínseca de crecimiento de la biomasa ( $\mathrm{r}$ $=0,75)$ y la capacidad de carga del recurso forrajero $\quad(\mathrm{K}=6385,39 \quad \mathrm{~kg} . \mathrm{MS} / \mathrm{ha} / \mathrm{mes})$ (Figura 1).

$$
C t=0,75^{*} Z t^{*}[1-(\mathrm{Zt} / 6385,39)]
$$

La biomasa forrajera que parte con un nivel inicial de 281,20 kg/MS/ha/mes, alcanza $\mathrm{K}$ en un periodo aproximado de 20 meses, siempre y cuando no exista la presencia de algún tipo de perturbación. Se puede indicar que, conforme se incrementa el nivel de stock del recurso forrajero el crecimiento alcanza un pico máximo de $1198,73 \mathrm{~kg} / \mathrm{MS} /$ ha/mes $(\mathrm{Zt}=3192,69 \mathrm{~kg} / \mathrm{MS} / \mathrm{ha} / \mathrm{mes})$ luego decae conforme biomasa se acerca a K. El máximo crecimiento de la biomasa forrajera estuvo por debajo de los valores promedios encontrados por Naupari (2000) y Esponda (2007) con 1398,55 kg/MS/ha/mes y $1413,72 \mathrm{~kg} / \mathrm{MS} / \mathrm{ha} / \mathrm{mes}$, respectivamente. Sin embargo, el crecimiento estimado en este trabajo es mayor a los 903, 851 y 745 $\mathrm{kg} / \mathrm{MS} / \mathrm{ha} / \mathrm{mes}$ reportados por Candelario (2005), Flores et al. (2005) y Avalos (2006)

\section{Función de consumo animal}

La función de consumo animal estimada fue significativa $(p<00,5)$. Se calculó el valor de $\mathrm{q}$ en 0,09350 y junto a los parámetros de la función de crecimiento $(\mathrm{a}=0,7509213$ y $b=0,0001176)$ y en base a la condición de equilibrio en estado estacionario, la función de consumo animal resultante que depende de la carga animal y se expresa de la siguiente forma:

$$
Y t=587,0504 \mathrm{Ca}-74,3431 \mathrm{Ca}^{2}
$$

A partir de esta función, se puede

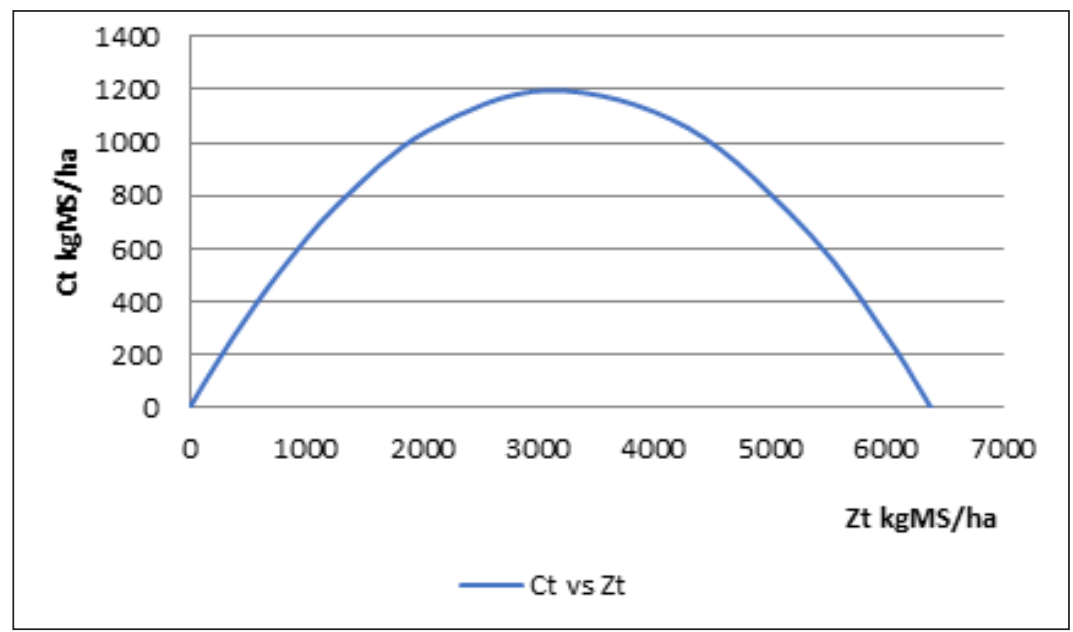

Figura 1: Crecimiento del recurso respecto al nivel de biomasa disponible 
conocer el nivel de carga animal en el punto de máximo rendimiento sostenible $(4,02$ $\mathrm{UAM} / \mathrm{ha}$ ) y también su respectivo nivel de consumo (1198,73 Kg.MS/ha/mes) (Figura 2).

Sin embargo, dado, dado que el nivel de consumo depende a su vez del nivel de biomasa disponible esta misma función se puede expresar de la siguiente manera:

$$
Y t=0,5677312 Z t-0,0000686 Z t
$$

El nivel de biomasa disponible donde se cumple la condición de sostenibilidad fue de $3738,57 \mathrm{Kg}$. MS/ha/mes, la cual le corresponde un nivel de carga animal de 3,33 UAM/ha y un nivel de consumo animal de $1163,69 \mathrm{Kg}$. MS/ha/mes (Figura 3).
La función de producción de leche alcanza un pico de producción a los 471,38 litros de leche con un nivel de carga animal igual a 4,02 UAM/ha. En el mismo sentido con un nivel de carga animal de 3,33 UAM/ mes le corresponde un nivel de producción de leche de 457,60 litros.

\section{Función de Ingresos y Costos}

Conocido los niveles de producción asociados a su respectiva carga animal, se procede a estimar los ingresos y costos totales de la actividad ganadera al pastoreo, con lo cual se puede calcular los beneficios de la actividad. Ambas funciones fueron significativas $(p<0,005)$ y su respectivo comportamiento se muestran en la Figura 4.

De acuerdo con la propiedad de maximización de los beneficios, se sabe

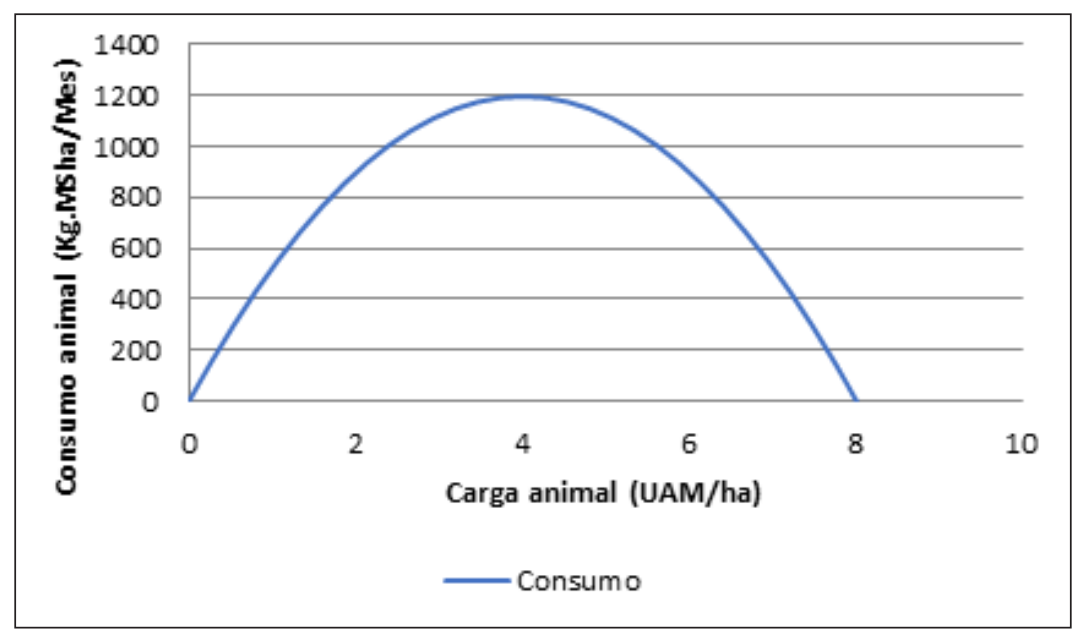

Figura 2: Funciones de consumo animal sostenible

\section{Función de Producción de Leche}

Representa la cantidad de leche producida en un mes por hectárea, que depende del nivel del consumo animal, la cual fue significativa $(\mathrm{P}<0,05)$ y que a la vez depende de la carga animal (Jones y Sandland, 1974).

$$
L=234,7785 C a-29,2340 C a^{2}
$$

que el ingreso marginal es igual al costo marginal $(\mathrm{IMg}=\mathrm{CMg})$ en el punto óptimo, con lo cual se determina el nivel de carga animal correspondiente a ese punto $\left(\mathrm{Ca}^{*}\right)$, es decir, el nivel de carga animal que nos brinda el Máximo Rendimiento Económico (MRE) de la actividad, el cual se estimó en 2,26 $\mathrm{UAM} / \mathrm{ha}$, así como también sus respectivos niveles de consumo animal, producción de leche y biomasa disponible estimados en $970 \mathrm{~kg} / \mathrm{MS} / \mathrm{ha} / \mathrm{mes}, 381,43$ Litros de leche y $4587,32 \mathrm{~kg} / \mathrm{MS} / \mathrm{ha} / \mathrm{mes}$, respectivamente. 


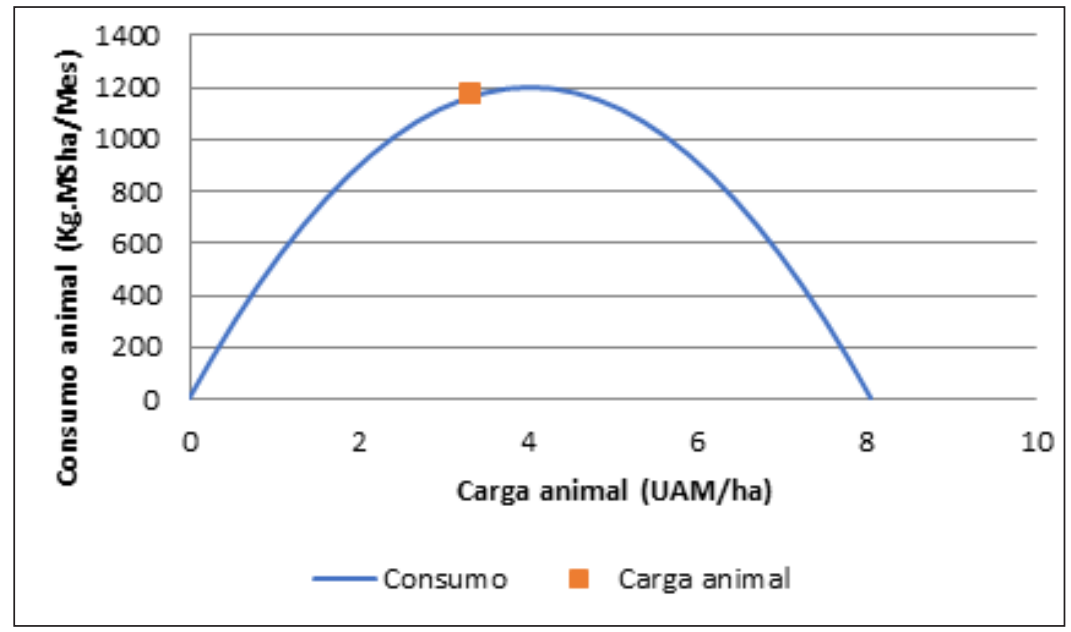

Figura 3: Nivel de carga animal

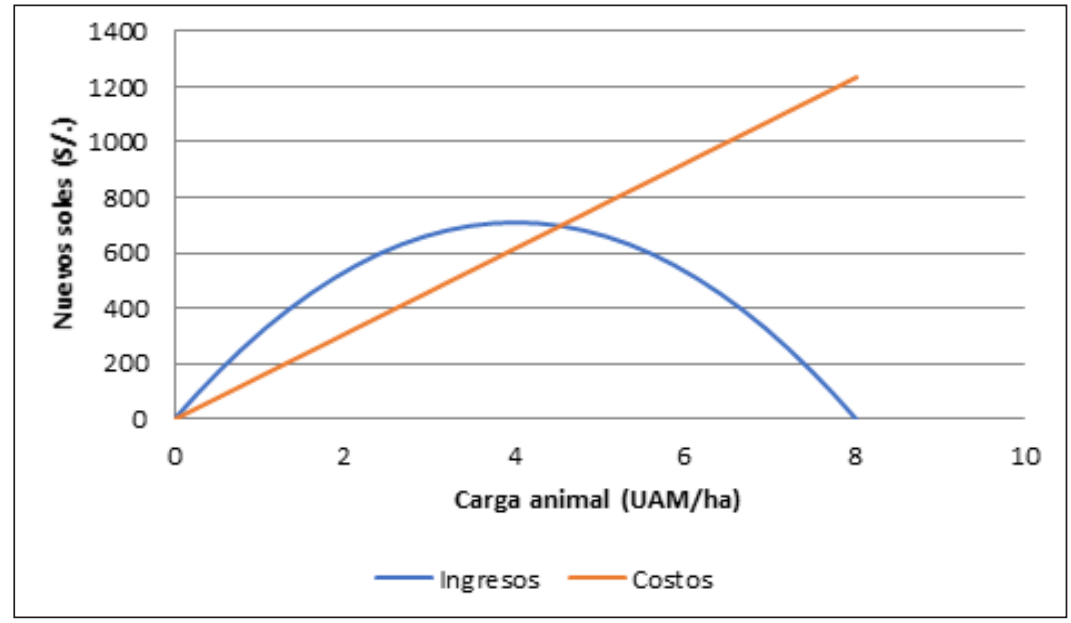

Figura 4: Funciones de ingresos y costos totales

De acuerdo con la propiedad de maximización de los beneficios, se sabe que el ingreso marginal es igual al costo marginal $(\mathrm{IMg}=\mathrm{CMg})$ en el punto óptimo, con lo cual se determina el nivel de carga animal correspondiente a ese punto $\left(\mathrm{Ca}^{*}\right)$, es decir, el nivel de carga animal que nos brinda el Máximo Rendimiento Económico (MRE) de la actividad, el cual se estimó en 2,26 $\mathrm{UAM} / \mathrm{ha}$, así como también sus respectivos niveles de consumo animal, producción de leche y biomasa disponible estimados en $970 \mathrm{~kg} / \mathrm{MS} / \mathrm{ha} / \mathrm{mes}, 381,43$ Litros de leche y $4587,32 \mathrm{~kg} / \mathrm{MS} / \mathrm{ha} / \mathrm{mes}$, respectivamente.

\section{Análisis de los beneficios}

Con la carga animal óptimo se calculó los beneficios económicos de la actividad ganadera en un sistema de producción de leche en base a una pastura. Podemos señalar que este nivel fue de 2,26 UAM/ha con S/ 
224,26 soles. Decisiones de incrementar la carga animal por sobre este nivel, implica reducción de beneficios hasta el punto donde estos se hacen nulos de 4,52 UAM/mes. Por la información obtenida en el presente estudio, se sugiere que el rango de carga animal donde debiéramos permanecer es entre 1,83 a 3,33 UAM/ha, sugiriendo de forma práctica que el rango donde debemos estar para hacer uso del recurso de forma racional y tener beneficios económicos es de 2 a 3 UAM/ha.

\section{Conclusiones}

El crecimiento de la biomasa presenta una tasa de crecimiento de 0,75 y una capacidad de carga de 6385,39 $\mathrm{Kg} / \mathrm{MS} / \mathrm{ha} / \mathrm{mes}$, capacidad que se alcanza sin la presencia de intervención humana. La biomasa alcanza un máximo de crecimiento de $1198,73 \mathrm{Kg} /$ $\mathrm{MS} / \mathrm{ha} / \mathrm{mes}$ cuando la disponibilidad es de $3192,60 \mathrm{~kg} / \mathrm{MS} / \mathrm{ha} / \mathrm{mes}$.

El nivel de consumo animal óptimo, en función de la información extraída de los estudios de perfiles alimentarios, fue de $970 \mathrm{~kg} / \mathrm{MS} / \mathrm{ha} / \mathrm{mes}$ al que le corresponde un nivel de carga animal de 2,26 UAM/ha, la cual maximiza los beneficios de la actividad ganadera en base a pasturas dedicada a la producción de leche.

El nivel óptimo de la carga animal de 2,26 $\mathrm{UAM} / \mathrm{ha} / \mathrm{mes}$ presenta un nivel de benéfico equivalente a de $\mathrm{S} / 224,26$, mientras que el nivel del máximo biológico de 4,02 UAM/ ha/mes fue de $\mathrm{S} / 89,35$ soles.

\section{Literatura citada}

ASRM [American Society Of Range Management And Agriculture Board]. 1962. Basic Problems and Techniques in Range Research. Report of a Joint Comitte of the American Society of Range management and Agriculture Board. Pub. No. 890. Washington D.C.344p.

Avalos, P. 2006. Dinámica de la producción forrajera y perfil alimenticio de vacas lecheras al pastoreo en un asociación gramínea - leguminosa en la sierra central. Tesis para optar el grado de Magister en Producción Animal, Universidad Nacional Agraria La Molina, Lima, Perú.
Bromley, D.W.1991. Environment and Economy: Property Rights and Public Policy. Oxford University Press. Oxford. United Kingdom. $247 \mathrm{p}$.

Caffera, M. 2018. Economía Ambiental Intermedia - Notas de Clase. Uruguay. Disponible en http:// www2.um.edu.uy/marcaffera/ docencia/Econom $\% \mathrm{C} 3 \% \mathrm{ADa} \% 20$ Ambiental\%20Intermedia/Notasde-clase-Economia-AmbientalIntermedia-Libro2018.pdf

Canavese, A. 2007. Temas en el Análisis Económico de los Derechos de Propiedad. Revista de Economía Política de Buenos Aires 1: 31-36.

Candelario, J. 2005.Valor nutritivo de la dieta, ingesta de forraje y perfil alimentario en vacas lecheras Brown Swiss Criollo en una pastura asociada de Dactylis - Trébol en secano en la sierra central. Tesis para optar el grado de Magister en Producción Animal, Universidad Nacional Agraria La Molina, Lima, Perú.

Conrad, J. 2010. Resource Economics Second Edition. Cambridge University Press. 300p.

Esponda, R. 2007. Productividad, composición química y capacidad de carga de una asociación Dactylis glomerata - Trifolium pratense en secano. Tesis para optar el grado de Magister en Producción Animal, Universidad Nacional Agraria La Molina, Lima, Perú.

Florez, A.; Malpartida, E. 1987. Manejo de praderas nativas y pasturas en la región altoandina del Perú. Tomo I. Fondo del libro del Banco Agrario. Lima, Perú.

Florez, A.; Bryant, F. 1990. Manual de pastos y forrajes. Dirección General de Investigación Pecuaria. Programa de Investigación de Pastos y Forrajes. Lima, Perú.

Flores, E.; Cruz, J.; Ñaupari, J. 2005. Utilización de praderas cultivadas en secano y praderas naturales para la producción lechera. Boletín Técnico CICCA-FDAINCAGRO. Lima, Perú. 
Flores, D. 2006. Producción primaria y flujo de energía en praderas naturales de Festuca-Calamagrostis y cultivadas de Dactylis-Trebol. Tesis para optar el grado de Magister en Producción Animal, Universidad Nacional Agraria La Molina, Lima, Perú.

Galarza, E. 2004. Economía de los Recursos Naturales. Universidad del Pacifico. Lima, Perú. 296p.

Galarza, E.; Collado, N. 2013. Los derechos de pesca: el caso de la pesquería de anchoveta peruana. Apuntes. Revista de Ciencias Sociales 40(73):7-42.

Gordon, H. 1954. Economic theory of a common-property resources: the fishery. J. Pol. Econ. 62: 129 - 142. Reprinted in Bull. Math. Biol. 53 (1/2): 231-252.

Hardin, G. 1968. The Tragedy of Commons. Science 162: 1243-1248.

INEI DE $\begin{aligned} & \text { [INSTITUTO NACIONAL } \\ & \text { ESTADISTICA }\end{aligned}$ IINFORMATICA]. 1994. III Censo Nacional Agropecuario - Resultados Definitivos. Informe técnico. Lima, Perú. 70p.

INEI ${ }_{\text {DE }}^{\text {[INSTITUTO NACIONAL }}$ IINFORMATICA]. 2012. IV Censo Nacional Agropecuario, Resultados Definitivos. Informe técnico. Lima, Perú. 63p.

INEI ${ }_{\text {DE }}^{\text {[INSTITUTO NACIONAL }}$ IINFORMATICA]. 2019. Evolución de la Pobreza Monetaria 2007-2018. Informe técnico. Lima-Perú. 181pp.

Jones, R.; Sandland, R. 1974: The relation between animal gam and stocking rate. The Journal of Agricultural Science 83 (02): 335-342.

L.“T MANNETJE”. 2001. Manejo de Pasturas Tropicales. CIAT. Colombia. Capítulo 3.

Noy-Meir, I. 1975. Stability of Grazing Systems: An Application of PredatorPrey Graphs. The Journal of Ecology 63(2): 459-481.

Maldonado, J. 2008. Economía de los Recursos Naturales: Aplicación de la Economía computacional a la solución de problemas Dinámicos. Universidad de los Andes, Facultad de Economía. Bogotá, Colombia. $74 \mathrm{p}$.

MEA [MILLENNIUM ECOSYSTEM ASSESSMENT]. 2005. Ecosystems and Human Well-being: Synthesis. Millennium Ecosystem Assessment. Island Press. Washington, DC.

MINAM [MINISTERIO DELAMBIENTE]. 2018. Mapa Nacional de Ecosistemas del Perú - Memoria Descriptiva. Ministerio del Ambiente del Perú. Lima, Perú. 117p.

Naupari， J. 2000. Comportamiento nutricional y perfil alimentario de vacas lecheras en pastos cultivados rye grass/trébol de la U.P. CONSAC. Tesis para optar el grado de Magister en Producción Animal, Universidad Nacional Agraria La Molina, Lima, Perú.

Ñaupari, J.; Flores, E. 1996. Análisis y diseño de planes de alimentación en pasturas. IV Congreso Nacional de Ingenieros Zootecnistas. Huancayo, Perú.

Oficina Nacional de Evaluacion de Recursos Naturales [ONERN]. 1985. Los Recursos Naturales del Perú. Lima, Perú. 325pp.

Ostrom, E. 2011. El gobierno de los comunes. La evolución de las instituciones de acción colectiva Segunda Edición. Instituto de Investigaciones Sociales. Universidad Nacional Autónoma de México. México. 395pp.

Riera, P.; García, D.; Kristrom, B.; Brannlund, R. 2005. Manual de Economía Ambiental y de los Recursos Naturales. Tercera Edición. Ediciones Paraninfo SA. Madrid. 356pp.

Ritten, J., Bastian, C., Frasier, W. 2010. Economically Optimal Stocking Rates: A Bioeconomic Grazing Model. Rangeland Ecology \& Management 63(4):407-414.

Romero, C. 1994. Economía de los Recursos Ambientales y Naturales Segunda Edición. Alianza Editorial. Madrid. 216pp.

Schaefer, M. 1954. Some aspects of the 
dynamics of population important to the management of the commercial marine fisheries. Bull. Inter-Am. Trop. Tuna Comm. 1 (2): 27-56. Reprinted in Bull. Math. Biol. 53(1/2): 253-279. 1991.

Seijo, J.; Defeo, O.; Salas, S. 1997. Bioeconomía Pesquera: Teoría Modelación y Manejo. Documento Técnico de Pesca. No $\mathrm{N}^{\mathrm{O}}$ 368. FAO, Roma. 176pp.

TEEB [THE ECONOMICS OF ECOSYSTEMS AND BIODIVERSITY]. 2010. The Economics of Ecosystems and Biodiversity Ecological and Economic Foundations. Edited by Pushpam Kumar. Earthscan, London and Washington.

Verhulst, P. 1838. Notice sur la loi que la population poursuit dans son accroissement. Correspondance mathematique et physique, 10, 113121.

Ward, F. A. 2006. Environmental and Natural Resource Economics First Edition. Pearson Prentice Hall. New Mexico State University. USA. 610pp. 